\title{
ON THE FORM OF A PLANE QUINTIC CURVE WITH FIVE CUSPS*
}

BY

PETER FIELD

§1. The equations of cuspidal tangents and the critical formulae.

It is well known that a quintic curve with 5 cusps can be obtained by inverting a quartic which is inscribed in and circumscribed about the fundamental triangle. The equation $\dagger$ of the quintic is:

$$
\begin{gathered}
\left(\alpha y^{2} z^{2}+\beta z^{2} x^{2}+\gamma x^{2} y^{2}-6 a x^{2} y z-6 b x y^{2} z-6 c x y z^{2}\right)^{2} \\
-(y z+z x+x y)^{3}\left(\alpha^{2} z y+\beta^{2} z x+\gamma^{2} x y\right)=0, \\
(\alpha=-a+b+c, \beta=a-b+c, \gamma=a+b-c),
\end{gathered}
$$

or more briefly

$$
C_{4}^{2}-\phi^{3} \phi_{1}=0 \text {. }
$$

Equation (1) is apparently of the eighth degree, but it contains the extraneous factors $x, y, z$. Dex Pezzo's paper gives no hint as to the possible forms of the curves represented by the above equation. In a paper $\ddagger$ upon quintic curves of deficiency 1 , I have given only one form of curve which could admit 5 real cusps. This, however, did not prove that only one such form existed ; for only those curves were there examined which were on the point of degenerating into a conic and cubic.

The problem which it is proposed to solve in the present paper is a purely geometrical one, viz., to obtain the general form of the curves represented by equation (1). For simplicity's sake $\phi$ will be taken a circle and the triangle of reference an equilateral triangle. $\S$ The figures were actually first obtained by drawing the conics $\phi, \phi_{1}$ and the quartic $C_{4}$, but these auxiliary curves will now be omitted, since the quintics can be drawn as well without their aid and the figures appear far more simple.

* Presented to the Society April 26, 1903. Received for publication Octobu 12, 1903, and February 20, 1905.

† See Dr. Pasquale dre Przzo in Rendiconti dell' Accademia delle soienze fisiche e matematiche (Sezione della societa reale di Napoli:), ser. 2, vol. 3 (1889), pp. 46-49.

$\$$ Quintic Curves for which $p=1$, A merican Journal of Matbematics, vol. 27 (1905).

\$ This is no restriction, as a conic through five given points can always be projected into a circle such that three of the given points are the vertices of an equilateral triangle. 
The following data which have been obtained directly from equation (1) will be used in drawing the curves. The cuspidal tangents (given incorrectly in DeL Pezzo's paper) at the vertices of the triangle of reference are:

$$
x=\frac{\alpha+c}{\beta+c} y, \quad x=\frac{\alpha+b}{\gamma+b} z, \quad y=\frac{\beta+a}{\gamma+a} z .
$$

The quintic passes through the points

$$
\begin{array}{ll}
x=0, & y=-\left(\frac{\alpha+c}{\alpha+b}\right)^{2} z, \\
y=0, & z=-\left(\frac{\beta+a}{\beta+c}\right)^{2} x, \\
z=0, & x=-\left(\frac{\gamma+b}{\gamma+a}\right)^{2} y
\end{array}
$$

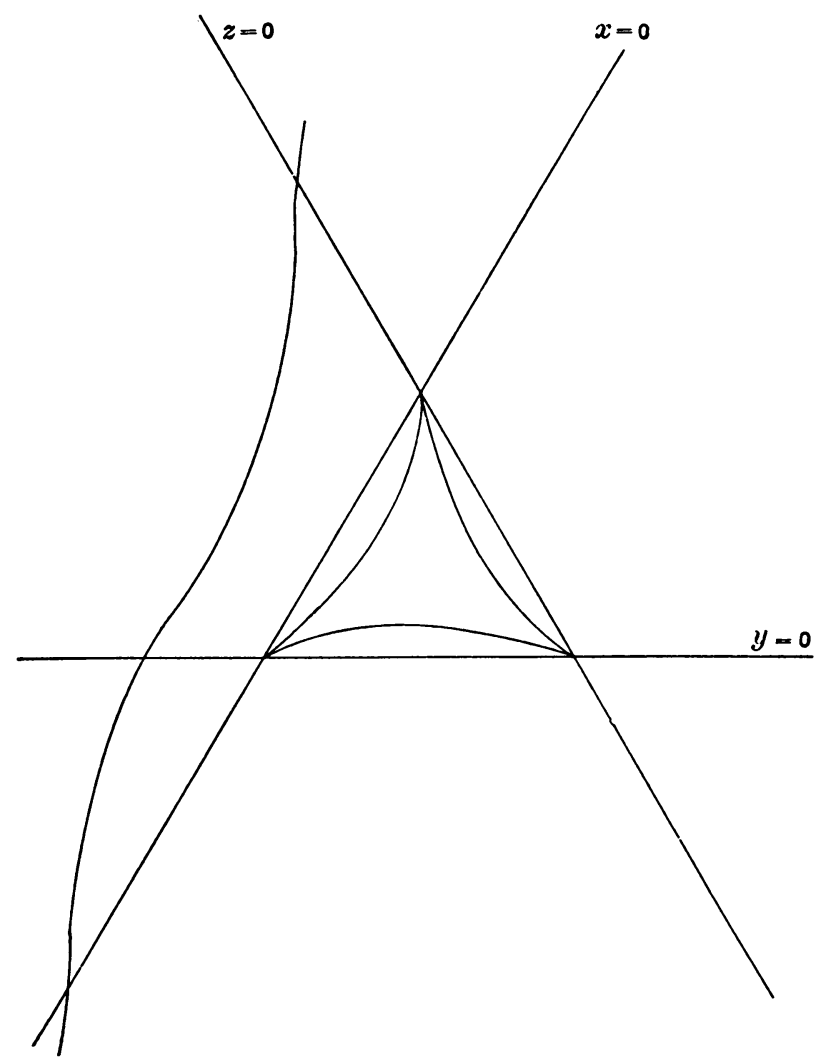

FIG. 1.

it has cusps also at the points where the lines $x=\left(-\gamma \pm \sqrt{\gamma^{2}-4 a b}\right) y / 2 a$ cut the circle which passes through the vertices of the fundamental triangle. 


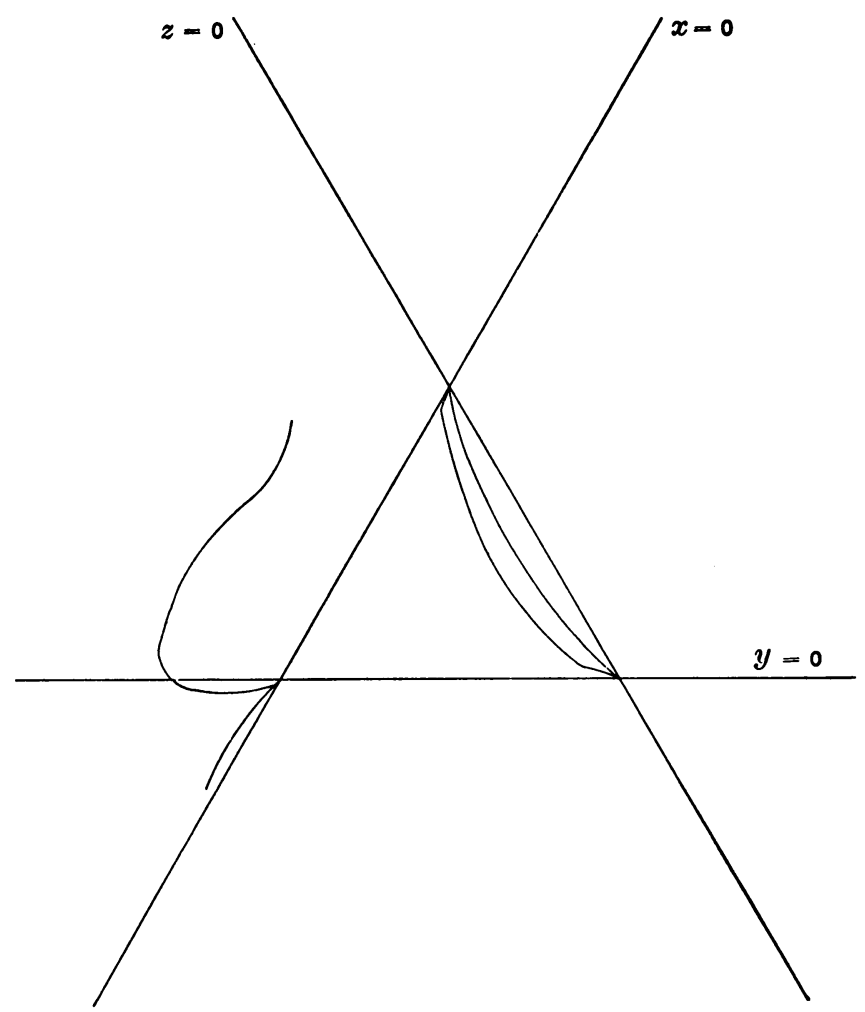

Fig. 2.

The direction of the curve at the points $x y, y z, z x$ must be such as to make the following expressions positive

$$
\begin{aligned}
& \frac{-256 c^{3} y^{3}}{(\beta+c)^{3}} \text { or } \frac{-256 c^{3} x^{3}}{(\alpha+c)^{3}}\left[a^{2}+b^{2}-2 a c-2 b c-a b+c^{2}\right], \\
& \frac{-256 c^{3} y^{3}}{(\beta+a)^{3}} \text { or } \frac{-256 a^{3} z^{3}}{(\gamma+a)^{3}}\left[c^{2}+b^{2}-2 a c-2 a b-b c+a^{2}\right], \\
& \frac{-256 b^{3} z^{3}}{(\gamma+b)^{3}} \text { or } \frac{-256 b^{3} x^{3}}{(\alpha+b)^{3}}\left[a^{2}+c^{2}-2 a b-2 b c-a c+b^{2}\right] .
\end{aligned}
$$

§ 2. Discussion of the formulce and application to diagrams.

First suppose $a>b>c$ and that they are all positive and nearly equal, then $\alpha, \beta, \gamma$ are all positive and $\alpha<\beta<\gamma$. From the given data it follows that Fig. 1 represents the given quintic. Two of the cusps are imaginary as long as $a<b+c+2 \sqrt{b c}$. As $a$ becomes nearly equal to $b+c$ the quintic may have the form given in Fig. 1 or it may be as represented in Fig. 2, because 
$a^{2}+b^{2}-2 a c-2 b c-a b+c^{2}$ may be either positive or negative according as $b \gtrless 3 c$, while at the two remaining vertices there is no such choice. The direction of the curve at the $z x$ vertex changes as $a$ passes through the value $b+\sqrt{3 b c-\frac{3}{4} c^{2}}+\frac{1}{2} c$ and at the $z y$ vertex it changes as $a$ passes through the value $b+\sqrt{\overline{3}} \overline{b c}+c$. Therefore if

$$
b+\sqrt{\overline{3} b \bar{c}}+c>a>b+\sqrt{\overline{3} b c-\frac{3}{4} c^{2}}+\frac{1}{2} c
$$

the corresponding curve will be as given in Fig. 3. As $a$ increases from $b+\sqrt{3 b c}+c$ to $b+2 \sqrt{b c}+c$ the circuit at $y z$ grows larger, crosses the line

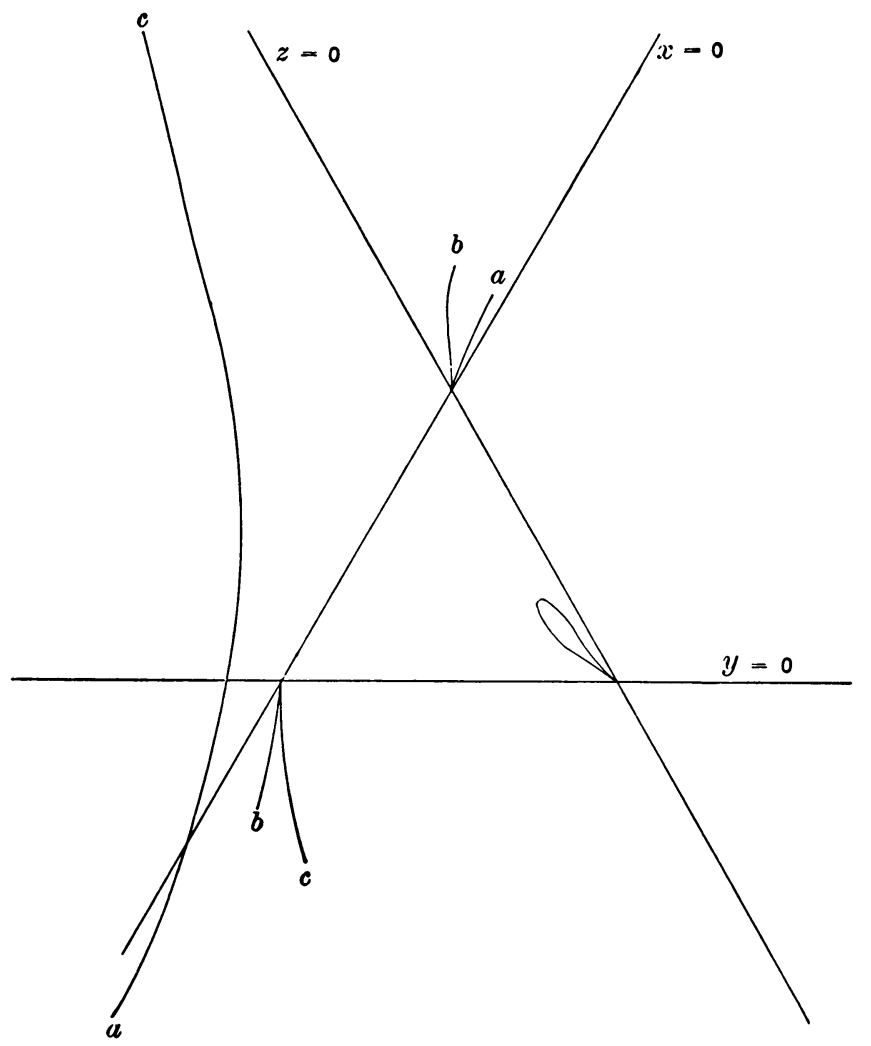

FIG. 3.

at infinity, and when $a=b+2 \sqrt{b c}+c$ it becomes tangent to the odd branch giving rise to a tacnode (Fig. 6 ) which is the transitional stage between three real and five real cusps. For values of $a$ greater than $(\sqrt{b}+\sqrt{c})^{2}$ the curve remains a single circuit with five real cusps. The curve is given in Fig. 4. This disposes of the case where $a, b, c$ have the same sign. The discussion for the case where they do not have the same sign will be omitted, as no new curves appear. 
In order to consider the case of four imaginary cusps it is convenient to modify equation (1) by replacing $x, y, a, b$ by $x+i y, x-i y, a+i b, a-i b$ respectively. This replaces the cuspidal points at $(x z)$ and $(y z)$ by cusps at the circular points. The equation then becomes

$$
\begin{array}{r}
{\left[(2 a-c)\left(x^{2}+y^{2}\right)^{2}-12 z\left(x^{2}+y^{2}\right)(a x-b y)-z^{2}\left(4 c x^{2}+8 b x y+8 c y^{2}\right)\right]^{2}} \\
-\left(x^{2}+y^{2}+2 z x\right)^{3}\left[(2 a-c)^{2}\left(x^{2}+y^{2}\right)+2 z\left(c^{2} x-4 b^{2} x-4 b c y\right)\right]=0 .
\end{array}
$$

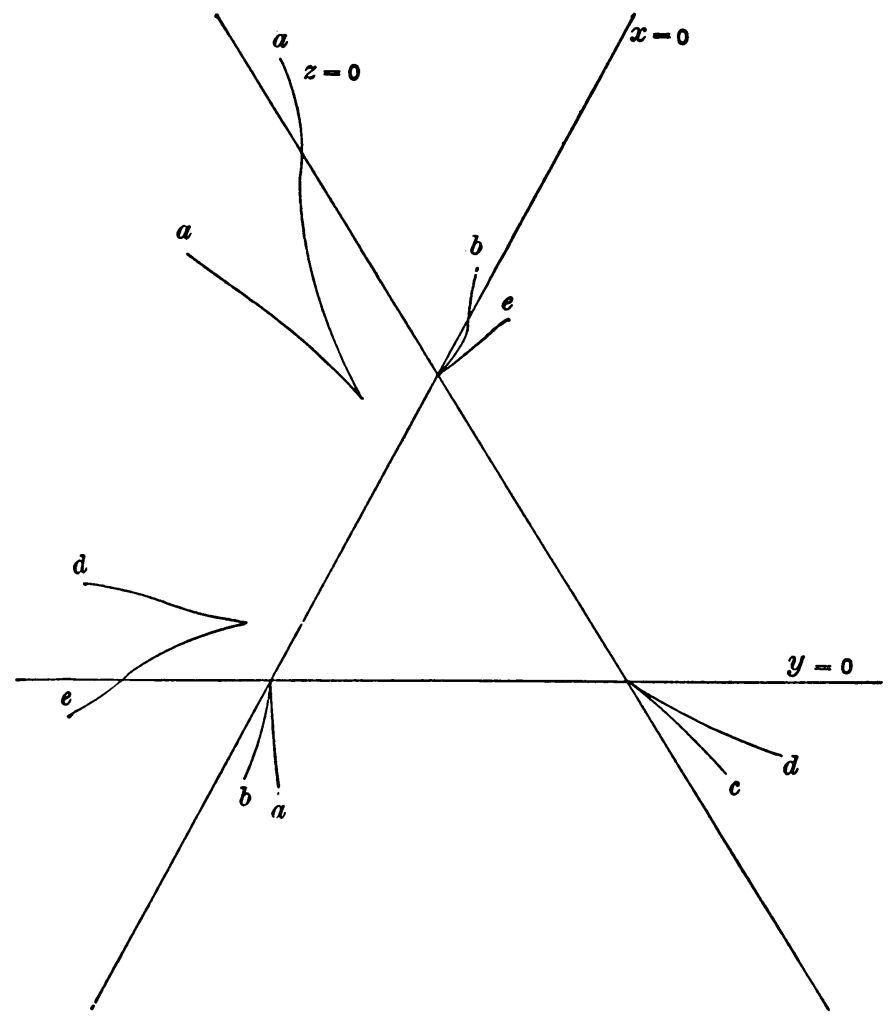

Fig. 4.

Equation (8) represents a curve with four imaginary cusps in case $4 b^{2}+4 a c-c^{2}<0$. The curve is given in Fig. 5 .

PLÜCKER's numbers for the curve under consideration are $m=n=k=i=5$, $\delta=\tau=0$. It follows from KLeIN's * equation, $n+w^{\prime}+2 t^{\prime \prime}=k+r^{\prime}+2 d^{\prime \prime}$, as well as from the figures that the number of real cusps is equal to the number of real inflexions. It is interesting to notice that each of the given classes of

* KLEIN, Eine neue Relation zwischen den Singulariłäten einer algebraischen Curve, Matbematische Annalen, vol. 10 (1876), p. 199. 
curves is its own dual. In Figs. 1 and 3 the even circuits go into odd circuits and the odd into even, while in Fig. 2 the reverse is the case. Fig. 4 has 5 real cusps and 5 real inflexions which occur alternately and the same would there-

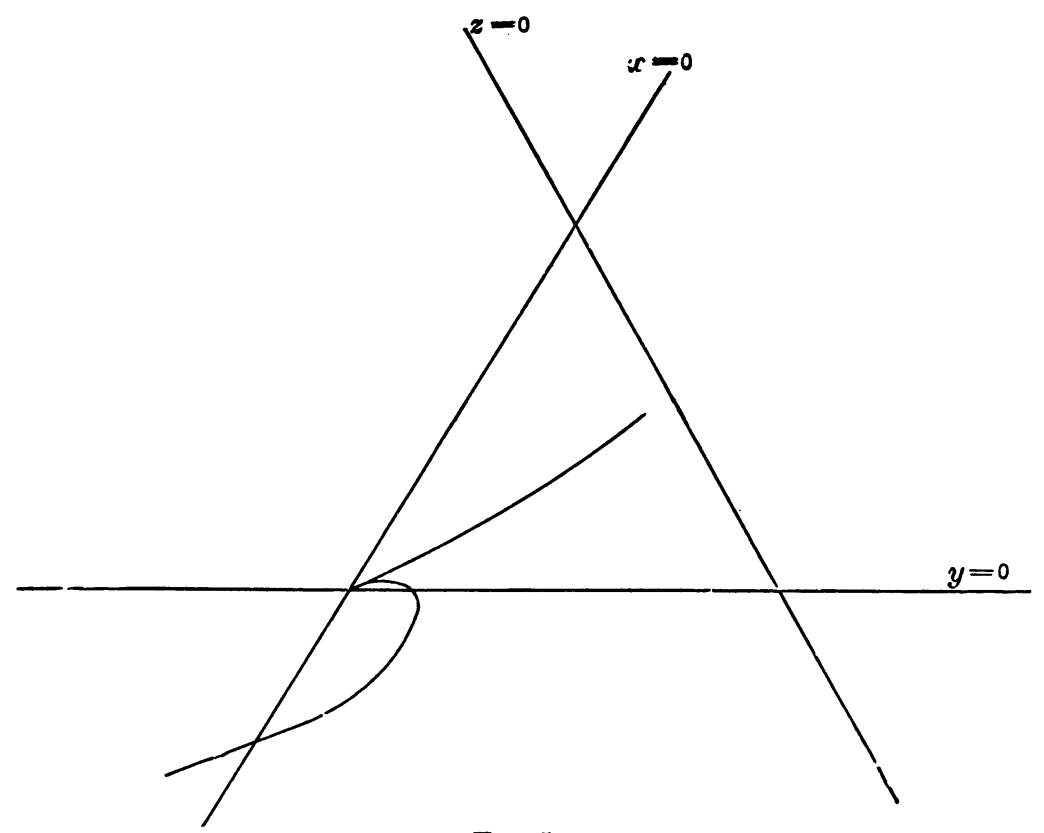

FiG. 5.

fore be the case with its dual. Fig. 5 has an odd circuit with one real cusp and one real inflexion and the same is true of its dual. The figures would therefore suggest that a curve and its dual may be projective.

The study of the 5 cusped quintic might also be connected with the study of a pentad of points in the plane.* If in addition to taking three of the cusps at the vertices of the triangle of reference, the fourth cusp were taken at the center of this triangle, the fifth cusp could be taken as the parameter of the system and it would be sufficient to study the curves in which this fifth cusp was located in any one of the 120 regions determined by the four fixed points. The coördinates of the fifth cusp would then be

$$
\left(1: \frac{\gamma-\sqrt{\gamma^{2}-4 a b}}{\gamma+\sqrt{\gamma^{2}-4 a b}}: \frac{\beta+\sqrt{\gamma^{2}-4 a b}}{\beta-\sqrt{\gamma^{2}-4 a} \bar{b}}\right) .
$$

* E. H. Moore, The Cross-ratio Group of $n$ ! Cremona Transformations of Order $n-3$ in Flat Space of $n-3$ Dimensions, A merican Journal of Mathematics, vol. 22 (1900). H. E. Slaugrt, The Cross-ratio Group of 120 Quadratic Cremona Transformations of the Plane, A merican Journal of Mathematics, vols. 22 and 23 (1901). 


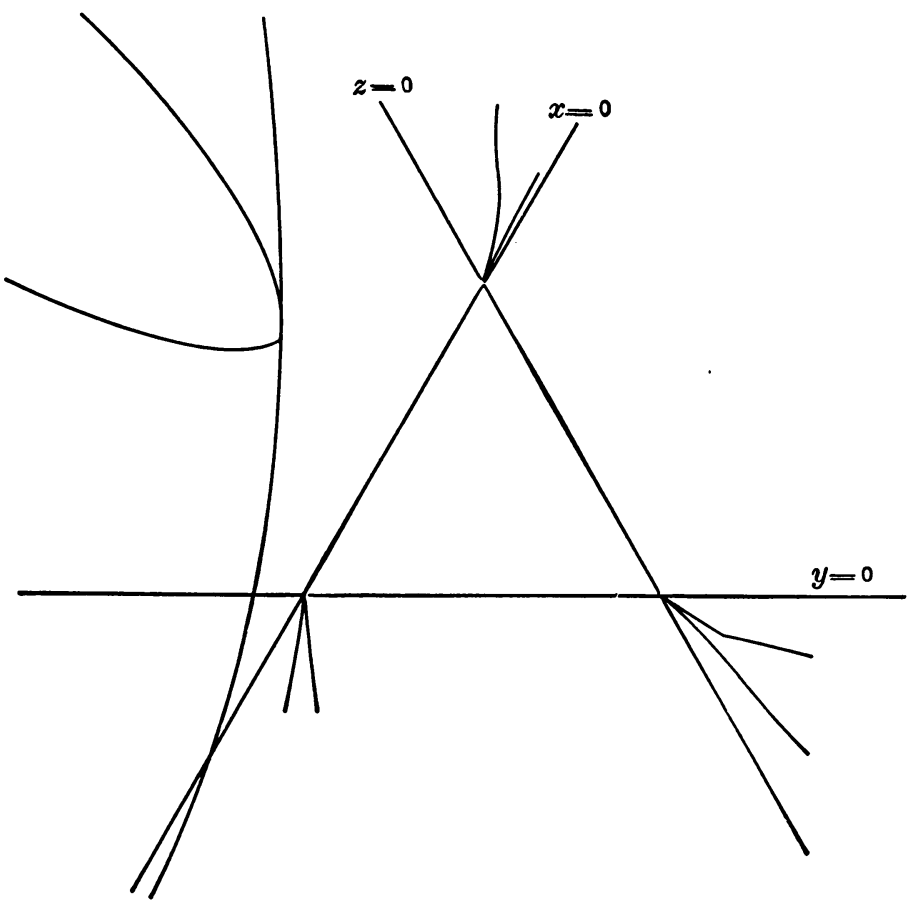

Fra. 6.

If

$$
\frac{\gamma-\sqrt{\gamma^{2}-4 a b}}{\gamma+\sqrt{\gamma^{2}-4 a b}}=\gamma_{1} \quad \text { and } \quad \frac{\beta+\sqrt{\gamma^{2}-4 a b}}{\beta-\sqrt{\gamma^{2}-4 a b}}=\gamma_{2} \text {, }
$$

$a: b: c$ can be determined in terms of $\gamma_{1}$ and $\gamma_{2}$, and any homogeneous relation between $a, b, c$ can be expressed in terms of $\gamma_{1}$ and $\gamma_{2}$.

ANN ARBOR, MICH., October 10, 1903. 